\title{
Perfil, atuação e satisfação de cirurgiões-dentistas em Residências Multiprofissionais em Saúde da região Sul do Brasil
}

\author{
Ana Marcia Mieko Yamaoka Oshima*; João Felipe Besegato**; Carolina Veloso Lima***; Mayara dos \\ Santos Noronha ${ }^{* * * * ;}$; Márcio Grama Hoeppner*****; Pablo Guilherme Caldarelli******* \\ * Estudante, Residência Multiprofissional em Saúde da \\ Família, Universidade Estadual de Londrina \\ ** Estudante, Programa de Pós-Graduação em Odontologia, \\ Universidade Estadual de Londrina \\ *** Estudante, Programa de Pós-Graduação em Odontologia, \\ Universidade Estadual de Campinas \\ **** Estudante, Programa de Pós-Graduação em Clínica \\ Odontológica, Universidade Estadual de Campinas \\ ***** Docente, Departamento de Odontologia Restauradora, \\ Universidade Estadual de Londrina \\ ****** Docente, Departamento de Medicina Oral e Odontologia \\ Infantil, Universidade Estadual de Londrina
}

Recebido em 11/07/2017. Aprovado em 26/11/2017.

\begin{abstract}
RESUMO
As Residências Multiprofissionais em Saúde (RMS) encontram-se distribuídas por todo o território nacional e estão estruturadas em abordagem multiprofissional com uma visão mais humana e abrangente, cujo foco não se restringe apenas aos procedimentos curativos. O objetivo do presente estudo foi avaliar o perfil dos cirurgiões-dentistas regularmente matriculados em RMS da região Sul do país, bem como sua atuação e satisfação com os programas. Para isso, foi realizada uma pesquisa quantitativa, exploratória e descritiva, utilizando-se um questionário on-line, cujo link foi enviado a cirurgiões-dentistas matriculados em RMS (Atenção Básica, Saúde da Família e Comunidade e Saúde Coletiva), localizadas na região Sul do Brasil. Os dados coletados foram tabulados no Microsoft Excel $^{\circledR} \mathrm{e}$ tratados por estatística descritiva (frequência simples e relativa). Constatou-se que a maioria dos residentes era do gênero feminino (75\%) com graduação em IES privada (70\%), sem experiência profissional prévia (70\%) e com experiência acadêmica prévia em saúde pública (85\%). Para os dados referentes à RMS, a maioria relatou como motivo pela escolha da residência o interesse em atuar na atenção básica. Oitenta e cinco por cento dos dentistas relataram atuar em Unidades Básicas de Saúde. A maioria dos residentes mostrou-se satisfeito com a RMS (75\%) e a falta de capacitação dos docentes foi apontada como a principal fragilidade dos programas. $\mathrm{O}$ estudo possibilitou traçar um panorama do perfil, atuação e satisfação dos cirurgiões-dentistas inseridos em RMS da região Sul do Brasil. Os dados podem auxiliar na melhoria das práticas e organização das RMS com cirurgiões-dentistas, conduzindo melhor a atuação de residentes e docentes frente às principais fragilidades apontadas.

Descritores: Cirurgiões Dentistas. Saúde da Família. Internato e Residência.
\end{abstract}




\section{INTRODUÇÃO}

Durante muitos anos as práticas odontológicas no Brasil foram centradas no atendimento da demanda espontânea, com enfoque individual e abordagem técnica. A realização dessas práticas não se encontrava relacionada a um planejamento integrado das ações, estando voltada apenas para a lógica de mercado. Com o intuito de ampliar e qualificar a saúde bucal nos serviços públicos de saúde, no ano de 2004, foram estruturadas as diretrizes da Política Nacional de Saúde Bucal (PNSB) ${ }^{1}$.

Com isso, o país deveria garantir qualidade e resolutividade dos serviços odontológicos, por meio de uma rede de atenção básica articulada com toda a rede de serviços, assegurando a integralidade das ações de saúde bucal, tanto a nível individual como coletivo, abrangendo desde a promoção e prevenção até o tratamento e a recuperação da saúde da população ${ }^{1}$. Ademais, essa política prevê uma adequação no processo de trabalho que requer uma abordagem interdisciplinar e multi/interprofissional, na qual as Equipes de Saúde Bucal (ESB) devem interagir com profissionais de outras áreas, ampliando seu conhecimento e permitindo uma abordagem do indivíduo como um todo ${ }^{1}$. Contudo, os cirurgiões-dentistas ainda encontram dificuldades em conciliar a prática diária, cuja demanda por procedimentos curativos permanece muito alta, com as atividades multiprofissionais, onde se destaca a troca de conhecimentos e o aprofundamento das relações, com vistas à prevenção das doenças bucais e promoção da saúde bucal.

Diante dessa problemática, o modelo das Residências Multiprofissionais em Saúde (RMS) foi estruturado com o intuito de formar profissionais de saúde com um novo perfil. Essas RMS operam na perspectiva de integração profissional entre as diversas áreas da saúde, baseada em práticas de humanização e comprometimento com o saber e poder coletivo, além da capacidade de responder às reais necessidades de saúde dos usuários $^{2}$. Nessa perspectiva, é esperado que a inserção dos residentes nos serviços de saúde fortaleça a atuação do cirurgião-dentista junto à equipe e às redes de atenção à saúde, além de potencializar o vínculo e o compromisso com pacientes, famílias e comunidade ${ }^{3}$.

Em acréscimo, na perspectiva de um trabalho multi/interprofissional, a RMS visa a integralidade do cuidado e o gradativo abandono da visão curativista e individualista. Assim, as RMS surgem como uma importante estratégia para o rompimento de antigas práticas e a introdução de uma nova visão para os profissionais da Odontologia e de outras áreas da saúde, que tem por objetivo superar a segmentação do conhecimento e do cuidado em saúde ${ }^{3}$.

Diante do exposto, buscando-se aprimoramento e melhor direcionamento das ações realizadas em RMS, o objetivo do presente estudo foi avaliar o perfil dos cirurgiões-dentistas regularmente matriculados em RMS da região Sul do país, bem como sua atuação e satisfação com os programas.

\section{METODOLOGIA}

Trata-se de um estudo quantitativo, transversal, exploratório e descritivo, o qual foi desenvolvido com cirurgiões-dentistas matriculados em RMS de Instituições de Ensino Superior (IES) da região Sul do Brasil, alinhadas junto à Comissão Nacional de Residência Multiprofissional em Saúde (CNRMS), sob a Resolução n. ${ }^{\circ}$ 1, de 30 de janeiro de $2012^{4}$. Para o desenvolvimento do presente estudo, foram selecionadas as residências multiprofissionais alocadas junto à Câmara Técnica 3 (CT3) da CNRMS, a qual engloba a Atenção Básica, Saúde da Família e Comunidade e Saúde 
Coletiva.

O acesso à listagem de residências se deu por meio do portal do Ministério da Educação e Cultura (MEC), no qual se encontram todos os programas de residência do território brasileiro em atividade, em processo de elaboração, em avaliação pela câmara técnica ou homologadas ${ }^{5}$. Participaram do estudo apenas as residências multiprofissionais que estavam em atividade. Foram excluídas da lista todas as residências que não contemplassem a Atenção Básica, Saúde da Família e Comunidade e Saúde Coletiva (CT3) e que não tivessem cirurgiões-dentistas na composição das equipes multiprofissionais. Dessa forma, das 15 RMS encontradas na região Sul do Brasil, nove foram selecionadas para o estudo.

O projeto de pesquisa foi aprovado pelo Comitê de Ética em Pesquisa da Universidade Estadual de Londrina (CAAE: 57939816. 1.0000.5231) e a concordância dos participantes se deu por meio de assinatura do Termo de Consentimento Livre e Esclarecido (TCLE).

O estudo foi realizado por meio de um questionário em formato eletrônico para preenchimento on-line. $\mathrm{O}$ conteúdo do questionário foi dividido em três partes: a primeira era composta por questões de múltipla escolha referente ao perfil do cirurgião-dentista (idade, gênero, estado civil, formação em escola pública ou privada, tempo de formação, pósgraduação, experiência profissional e acadêmica em saúde pública); a segunda possuía questões referentes à atuação do residente na RMS (motivos que levaram o residentes a cursar a RMS, atuação com outras áreas profissioanais, local de atuação, períodos de atividades teóricas, práticas e em grupos); e uma terceira parte com questões sobre a satisfação do residente quanto à integração em equipe, às práticas realizadas e a organização da RMS, utilizando "escala Likert"6 e questões de múltipla escolha.
Para a seleção dos participantes, foram coletados os contatos (endereços de e-mail e telefones) dos coordenadores e/ou responsáveis pelas RMS de nove IES da região Sul, por meio dos sítios eletrônicos das instituições. Em seguida, foram enviadas mensagens eletrôncias que continham a descrição do projeto de pesquisa, a solicitação dos contatos de residentes matriculados e o parecer consubstanciado do Comitê de Ética em Pesquisa, para o esclarecimento de dúvidas e idoneidade ética da pesquisa. Além disso, contatos telefônicos foram realizados junto aos coordenadores e/ou responsáveis que não responderam às mensagens eletrônicas em um período de 20 dias.

Com a obtenção dos contatos dos residentes foi possível enviar e-mail com o link do questionário, ficando a critério de cada participante a concordância da participação. Para isso, o residente deveria acessar o link informado na mensagem eletrônica e na primeira tela do questionário, visualizar o TCLE e clicar na opção afirmativa para participação. Logo após essa etapa, o residente teria acesso ao questionário. Este questionário on-line foi elaborado no programa Google Drive ${ }^{\circledR}$, ficando disponível pelo período de 60 dias para preenchimento.

Os dados coletados por meio dos questionários foram tabulados no software Microsoft Excel $^{\circledR}$ e tratados por estatística descritiva (frequências simples e relativa).

\section{RESULTADOS}

Dentre as instituições pesquisadas, oito responderam e enviaram o contato de 24 residentes regularmente matriculados, dos quais 20 acessaram e responderam ao questionário. Dentre as oito Instituições participantes, seis (75\%) encontram-se vinculadas a IES públicas e $2(25 \%)$ a IES privadas. Os principais resultados do questionário referente ao perfil pessoal e profissional dos residentes participantes do 
estudo estão descritos nas tabelas 1 e 2 .

As figuras 1 e 2 apresentam, respectivamente, os motivos que levaram os cirurgiõesdentistas a cursarem a RMS e áreas profissionais das RMS que atuam conjuntamente com a Odontologia nos programas participantes do estudo.
As principais fragilidades das RMS apontadas pelos cirurgiões dentistas estão descritas na figura 3 .

O grau de satisfação dos cirurgiõesdentistas quanto às práticas e organização das RMS encontra-se apresentado na tabela 3.

Tabela 1. Perfil dos cirurgiões-dentistas regularmente matriculados em RMS da região Sul do Brasil participantes do estudo $(\mathrm{n}=20)$.

\begin{tabular}{|c|c|c|}
\hline Perfil & $\mathbf{n}$ & $\%$ \\
\hline \multicolumn{3}{|l|}{ Gênero } \\
\hline Masculino & 5 & $25 \%$ \\
\hline Feminino & 15 & $75 \%$ \\
\hline \multicolumn{3}{|l|}{ Idade } \\
\hline $20-25$ anos & 10 & $50 \%$ \\
\hline $26-30$ anos & 7 & $35 \%$ \\
\hline $31-35$ anos & 1 & $5 \%$ \\
\hline $41-60$ anos & 2 & $10 \%$ \\
\hline \multicolumn{3}{|l|}{ Estado civil } \\
\hline Casado & 3 & $15 \%$ \\
\hline Solteiro & 17 & $85 \%$ \\
\hline \multicolumn{3}{|l|}{ Formação (graduação) } \\
\hline Pública & 6 & $30 \%$ \\
\hline Privada & 14 & $70 \%$ \\
\hline \multicolumn{3}{|l|}{ Tempo de formação } \\
\hline $1-3$ anos & 14 & $70 \%$ \\
\hline $4-9$ anos & 4 & $20 \%$ \\
\hline Mais de 10 anos & 2 & $10 \%$ \\
\hline \multicolumn{3}{|c|}{ Pós-graduação (stricto elou lato sensu) } \\
\hline Sim & 6 & $30 \%$ \\
\hline Não & 14 & $70 \%$ \\
\hline \multicolumn{3}{|c|}{ Experiência profissional (prévia) em saúde pública } \\
\hline Sim & 6 & $30 \%$ \\
\hline Não & 14 & $70 \%$ \\
\hline \multicolumn{3}{|c|}{ Experiência acadêmica em saúde pública } \\
\hline Sim & 17 & $85 \%$ \\
\hline Não & 3 & $15 \%$ \\
\hline
\end{tabular}


Tabela 2. Atuação dos cirurgiões-dentistas em RMS da região Sul do Brasil participantes do estudo (n=20).

\begin{tabular}{lcc}
\hline Atuação & n & \% \\
\hline Local & 2 & $10 \%$ \\
Hospital & 17 & $85 \%$ \\
$\quad$ Unidade Básica de Saúde & 1 & $5 \%$ \\
$\quad$ Instituição de Ensino Superior & & $55 \%$ \\
Períodos* de prática clínica & 11 & $25 \%$ \\
1 a 3 períodos & 5 & $20 \%$ \\
4 a 6 períodos & 4 & \\
7 a 9 períodos & & $45 \%$ \\
Períodos* de atividades em grupo & 9 & $35 \%$ \\
1 a 2 períodos & 7 & $20 \%$ \\
3 a 4 períodos & 2 & \\
5 a 6 períodos & & $50 \%$ \\
Períodos* de atividades teóricas & 10 & $40 \%$ \\
1 a 2 períodos & 8 & $10 \%$ \\
3 a 4 períodos & 2 & \\
5 a 6 períodos & & \\
\hline
\end{tabular}

* Carga horária referente a cada período: 4 horas.

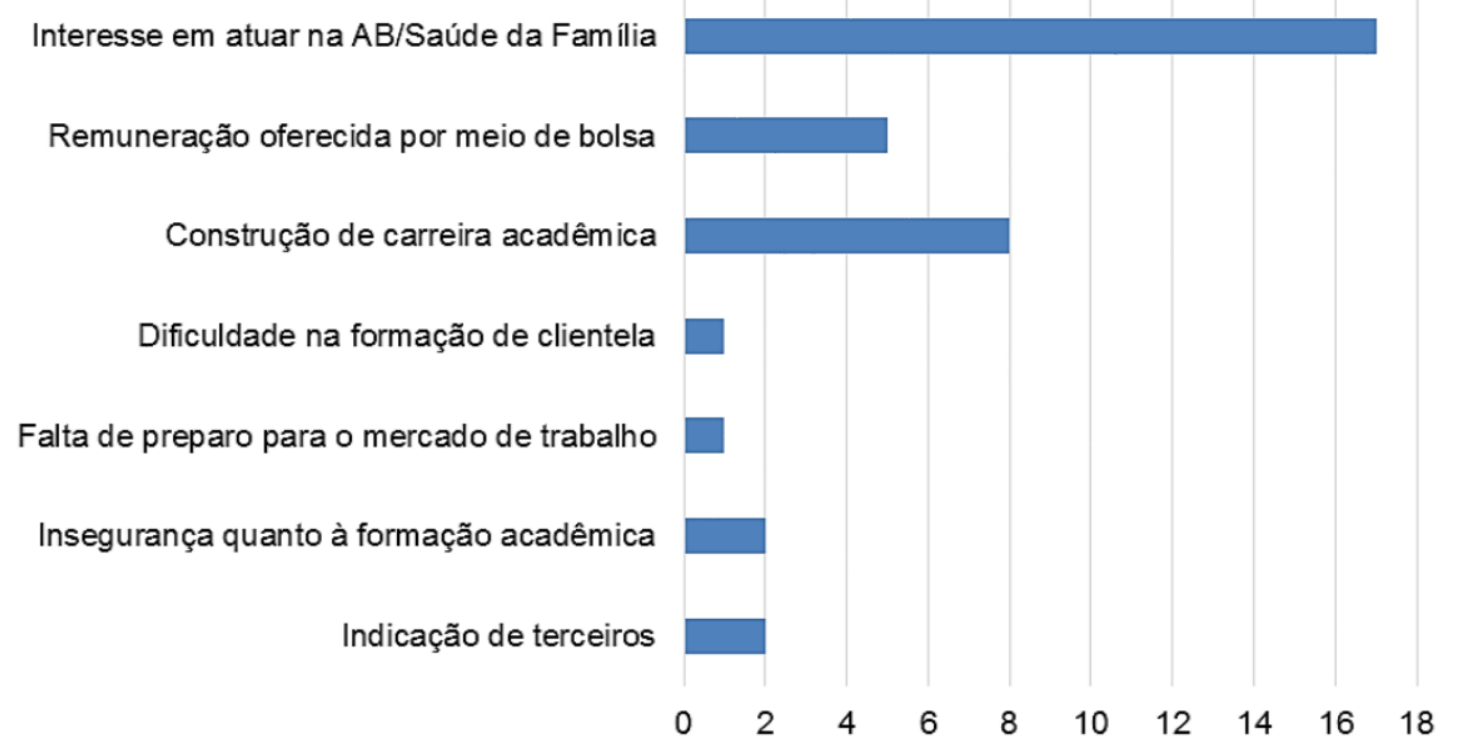

Figura 1. Motivos que levaram os cirurgiões-dentistas a cursarem as RMS da região Sul do Brasil participantes do estudo. 


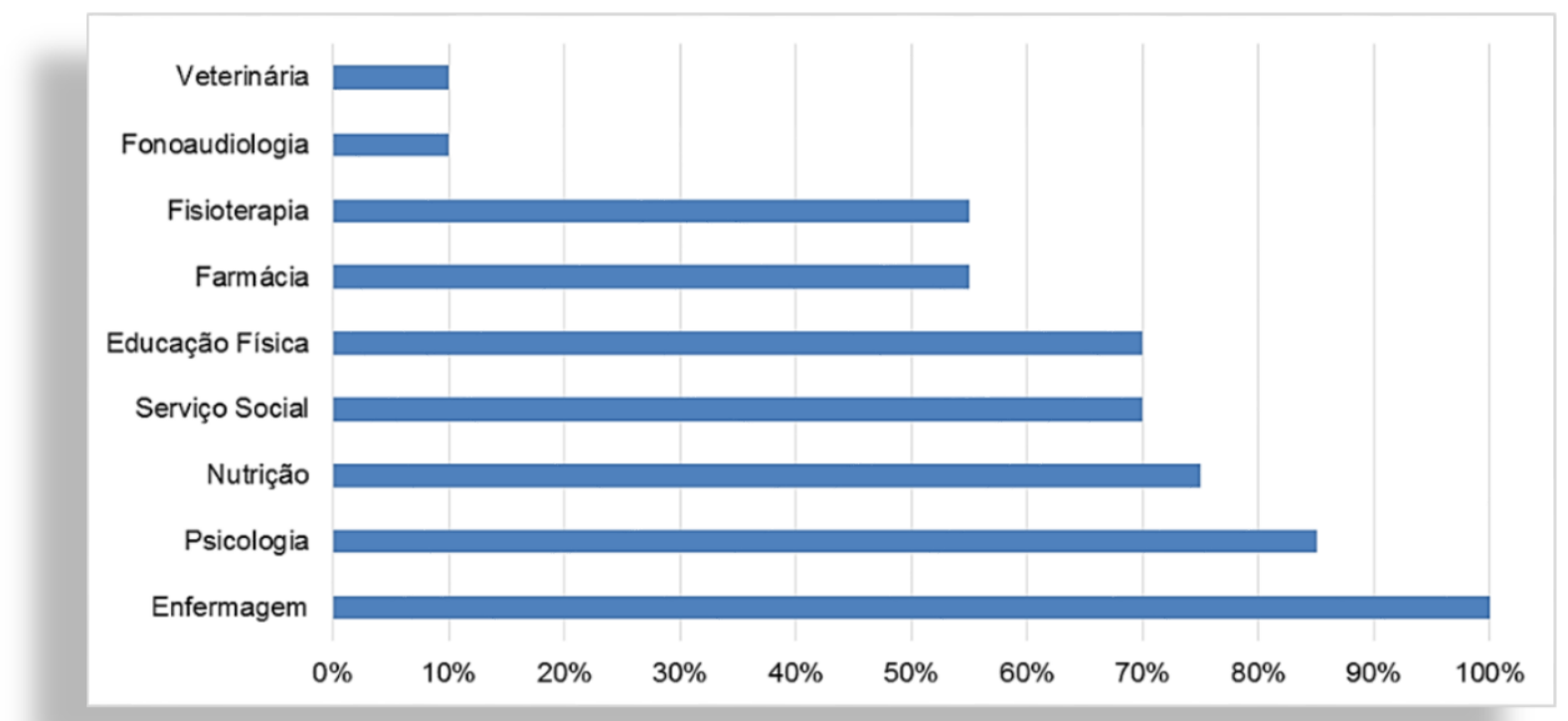

Figura 2. Áreas profissionais da RMS que atuam conjuntamente com a Odontologia nas RMS da região Sul do Brasil participantes do estudo.

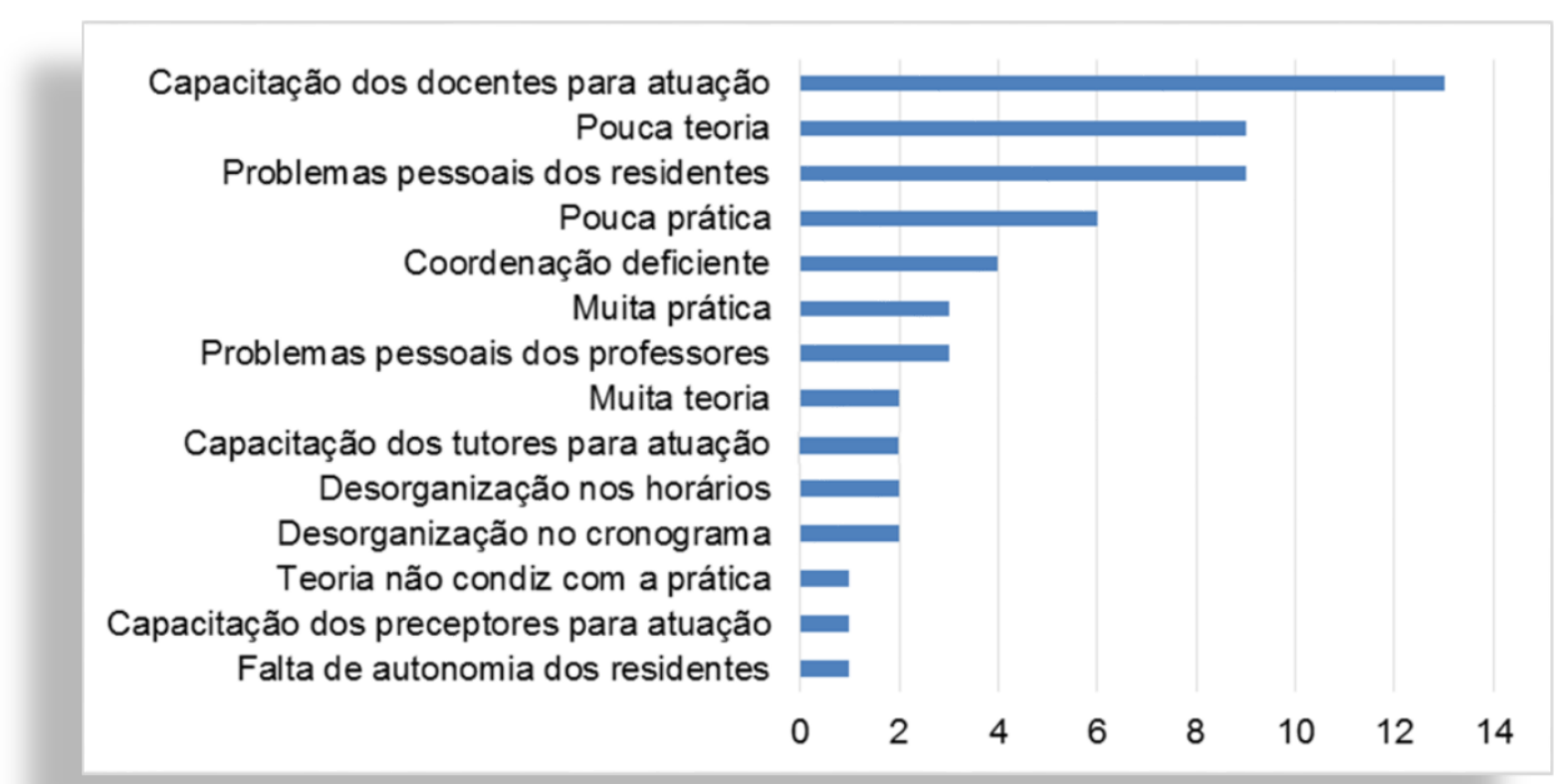

Figura 3. Principais fragilidades das RMS apontadas pelos cirurgiões dentistas participantes do estudo. 
Tabela 3. Grau de satisfação dos cirurgiões-dentistas quanto às práticas e organização das RMS da região Sul do Brasil participantes do estudo $(n=20)$.

\begin{tabular}{|c|c|c|c|c|c|c|c|c|c|c|}
\hline & \multicolumn{2}{|c|}{$\begin{array}{c}\text { Muito } \\
\text { insatisfeito }\end{array}$} & \multicolumn{2}{|c|}{ Insatisfeito } & \multicolumn{2}{|c|}{$\begin{array}{l}\text { Nem satisfeito, nem } \\
\text { insatisfeito }\end{array}$} & \multicolumn{2}{|c|}{ Satisfeito } & \multicolumn{2}{|c|}{$\begin{array}{c}\text { Muito } \\
\text { satisfeito }\end{array}$} \\
\hline & $\mathrm{n}$ & $\%$ & $\mathrm{n}$ & $\%$ & $\mathrm{n}$ & $\%$ & $\mathrm{n}$ & $\%$ & $\mathrm{n}$ & $\%$ \\
\hline Atuação na residência & 1 & $5 \%$ & - & - & 3 & $15 \%$ & 15 & $75 \%$ & 1 & $5 \%$ \\
\hline Interação entre residentes & 1 & $5 \%$ & - & - & 3 & $15 \%$ & 9 & $45 \%$ & 7 & $35 \%$ \\
\hline $\begin{array}{l}\text { Interação entre } \\
\text { profissionais }\end{array}$ & 1 & $5 \%$ & 2 & $10 \%$ & 6 & $30 \%$ & 8 & $40 \%$ & 3 & $15 \%$ \\
\hline $\begin{array}{l}\text { Interação entre equipe de } \\
\text { saúde bucal }\end{array}$ & 1 & $5 \%$ & - & - & 3 & $15 \%$ & 4 & $20 \%$ & 12 & $60 \%$ \\
\hline $\begin{array}{l}\text { Saúde Coletiva aplicada } \\
\text { no dia a dia }\end{array}$ & 2 & $10 \%$ & 3 & $15 \%$ & 7 & $35 \%$ & 7 & $35 \%$ & 1 & $5 \%$ \\
\hline $\begin{array}{l}\text { Formação acadêmica } \\
\text { como pré-requisito para } \\
\text { residência }\end{array}$ & 1 & $5 \%$ & 5 & $25 \%$ & 4 & $20 \%$ & 7 & $35 \%$ & 3 & $15 \%$ \\
\hline
\end{tabular}

\section{DISCUSSÃO}

Com relação ao perfil dos cirurgiõesdentistas matriculados nas RMS, destacou-se o sexo feminino, o profissional mais jovem, solteiro, recém-formado, com maioria tendo feito graduação em instituição privada, sem pósgraduação, sem experiência profissional em saúde pública e com experiência acadêmica em saúde pública. De acordo com o SEMESP $(2015)^{7}$, que é a $6^{\mathbf{a}}$ edição do Mapa do Ensino Superior, uma pesquisa realizada entre 2000 e 2013, o número de IES no Brasil teve um crescimento total de $102,6 \%$, sendo $71 \%$ nas públicas e 108,2\% nas privadas. Em 2013, havia 2391 instituições privadas e 301 públicas e isso poderia justificar o fato da maioria dos residentes serem formados em Instituições particulares.

A falta de pós-graduação e a pouca experiência profissional em saúde pública pode estar relacionada ao pouco tempo de formação da maioria dos residentes dos programas. Em relação aos estágios acadêmicos em serviços públicos de saúde pelos estudantes no processo de formação, ressalta-se que os mesmos são obrigatórios, considerando as Diretrizes Curri- culares Nacionais (DCN) dos cursos de graduação em Odontologia ${ }^{8}$. Dessa forma, os estágios permitem uma capacitação de recursos humanos para atuar no modelo assistencial vigente no país, que podem provocar sentimentos positivos ou negativos, mas construtivos para o estudante em formação9 .

Adicionalmente, dentre os motivos que levaram os cirurgiões dentistas a optarem pela RMS, as opções mais citadas foram o interesse na atuação em atenção básica/saúde da família, construção de carreira acadêmica e remuneração oferecida por meio da bolsa. $\mathrm{O}$ interesse pela atenção básica é fator favorável e promotor de um bom desenvolvimento no transcorrer das RMS, uma vez que para o serviço público, o cirurgião-dentista ideal é aquele que simpatiza com o coletivo, que é capaz de proporcionar um atendimento humanizado mesmo com pouca experiência e que principalmente tenha respeito aos aspectos éticos de sua profissão ${ }^{10}$. Além disso, de acordo com as $\mathrm{DCN}^{8}$, ressalta-se que o processo de formação dos cirurgiões dentistas deve estar embasado no desenvolvimento de um profissional humanista, crítico, reflexivo e capaz 
de atuar nos diversos cenários e níveis de atenção $^{8,11}$.

Quando se discute a carreira acadêmica e a remuneração, o estudo de Saliba et al. $(2012)^{12}$ constatou que há uma saturação de profissionais da Odontologia, o que torna difícil conseguir um emprego/local de trabalho logo ao sair da faculdade, sendo que muitas vezes as condições não são adequadas e a remuneração é baixa, fazendo com que esses profissionais optem pela residência devido a renda garantida e o contínuo aprendizado que passa a ser um diferencial, juntamente com o título de especialista. Destacase que o valor pago como bolsa de estudos aos residentes é maior que o piso salarial de muitas categorias da RMS, o que acaba sendo um fator decisivo na escolha de alguns.

Quanto aos profissionais que atuam conjuntamente com Odontologia nas RMS participantes desse estudo destacam-se: enfermeiros, psicólogos, nutricionista, assistentes sociais, profissionais da educação física, farmacêuticos, fisioterapeutas, fonoaudiólogos e médicos veterinários. Os resultados apontam que a enfermagem é a categoria profissional que aparece unânime em conjunto com todas as RMS, tratando-se de uma profissão fortemente inserida no $\operatorname{SUS}^{13}$ e que apresenta o maior número de residentes nos programas. Nesse cenário, destaca-se, ainda, que a participação das distintas profissões na RMS é dependente de vários fatores, como a existência ou não do curso na IES, a capacidade do curso na implementação e funcionamento da RMS, além da necessidade da gestão municipal em inserir determinada profissão dentro da proposta.

Segundo a Resolução $\mathrm{n}^{\circ} 5$ de 7 de novembro de 2014 da CNRMS $^{14}$, os programas de Residência têm duração mínima de dois anos, com carga horária mínima de 5.760 horas, totalizando 60 horas semanais. Dessa carga horária total, $80 \%$ devem ser destinadas às práticas e teórico-práticas, e 20\% para estratégias educacionais teóricas. Tendo em vista os resultados da carga horária prática que demonstram que $55 \%$ dos residentes tem apenas 1 a 3 períodos de prática clínica por semana (sendo cada período referente a 04 horas) podese dizer que, dificilmente, estes alunos conseguiriam alcançar os $80 \%$ das práticas exigidas pelo programa. Ressalta-se que isso pode interferir no processo de formação dos residentes e também na composição da escala semanal dos profissionais, inclusive na obrigatoriedade legal de plantões para o cumprimento da carga horária total.

Além disso, os resultados do presente estudo mostram que $85 \%$ das atividades práticas dos programas de RMS são desenvolvidas em Unidades Básicas de Saúde (UBS), as quais oferecem um serviço abrangente à população, considerando os aspectos sociais, psicológicos e biológicos de seus usuários ${ }^{15}$. Tal fato já era esperado, uma vez que as RMS selecionadas para o presente estudo encontram-se alocadas junto à Câmara Técnica 3 (CT3) da CNRMS ${ }^{4}$, a qual engloba a Atenção Básica, Saúde da Família e Comunidade e Saúde Coletiva.

Quanto à satisfação dos residentes, os resultados são bem diversificados, demonstrando opiniões divergentes quanto à atuação e interação entre os profissionais. Em relação à atuação nos programas de RMS, a grande maioria se mostra satisfeita ou muito satisfeita. Já com relação à integração entre os próprios residentes, observou-se determinado equilíbrio, estando a maioria satisfeita ou muito satisfeita. Esse fato é importante, pois a residência é uma experiência única e envolve um número seleto de pessoas, o que pode acabar trazendo uma pactuação entre os integrantes, que diante de novas experiências em conjunto, formam uma espécie de união, cujo vínculo se torna sólido no decorrer dos dois anos de duração da residência. 
No caso da integração entre residentes e os demais profissionais dos serviços públicos de saúde, apesar da maioria estar satisfeita ou muito satisfeita, uma parcela considerável se mostrou neutra. Para Alves et al. $(2014)^{16}$, as dificuldades na abordagem interdisciplinar e multi/ interprofissional podem estar relacionas às deficiências das matrizes curriculares dos cursos de graduação, o que resulta em ações isoladas de cada profissional, na sobreposição das ações de cuidado e sua fragmentação, dificultando esta interação entre os profissionais. Com relação à interação entre residente e a ESB a maioria relatou estar muito satisfeita, o que pode ser descrito como uma característica da profissão, pois o trabalho dentro da clínica odontológica exige sintonia: cirurgião-dentista, auxiliares e técnicos em saúde bucal se complementam durante o trabalho, o que tende a formar um forte vínculo.

Sobre a "Saúde Coletiva aplicada no dia a dia", a maioria dos cirurgiões-dentistas disse estar nem satisfeito nem insatisfeito. De acordo com Souza (2014) ${ }^{17}$, a Saúde Coletiva é uma área do saber que toma como objetivo as necessidades sociais de saúde entendendo a situação de saúde como um processo social relacionado à estrutura da sociedade e concebendo as ações de atenção à saúde como práticas simultaneamente técnicas e sociais, propondo intervenções articuladas de promoção, proteção, recuperação e reabilitação da saúde, baseadas em abordagem multidisciplinar. De acordo com os resultados encontrados no presente estudo, percebe-se que o cirurgião-dentista ainda tem seu trabalho centrado em atendimentos clínicos com uma visão curativista, não considerando o diagnóstico situacional no planejamento do tratamento dos seus pacientes. Segundo Nascimento et al. $(2006)^{18}$, a formação inicial da maioria dos profissionais de saúde (ainda) se baseia em um ensino tradicional tecnicista, com enfoque no indivíduo, descontextualizado e muitas vezes sem abertura para problematizar, refletir e construir um saber inerente à prática profissional. No entanto, destaca-se que o enfoque coletivo não pode ser deixado de lado em função da hegemonia do enfoque clínico.

Por fim, $50 \%$ dos dentistas se mostrou insatisfeito ou neutro quando foram questionados sobre a necessidade de formação acadêmica como pré-requisito para a residência, enquanto $50 \%$ indicou estar satisfeita ou muito satisfeita. Tais resultados podem apontar para dois horizontes: o fato de o ensino de graduação ser deficitário para a área de saúde pública ${ }^{19}$ e/ou ao fato de que as universidades possuem potencial para contribuir com a saúde por meio da criação de ambientes de trabalho, aprendizagem e vivências saudáveis para estudantes e funcionários, ampliando a importância da saúde pública no ensino e na pesquisa, além de desenvolver alianças e parcerias para a promoção da saúde e atuação comunitária ${ }^{20}$.

O maior problema apontado pelos residentes nos programas foi aquele relacionado à capacitação dos docentes para atuação nas RMS. Diante desse cenário, torna-se importante compreender a importância e o papel desempenhado por três distintos atores que integram as RMS e participam ativamente do processo ensino-aprendizagem: docentes, tutores e preceptores. Tutores e docentes estão ligados às IES, portanto, sendo necessária a titulação mínima de mestre ou doutor, já os preceptores estão vinculados às instituições executoras (serviços de saúde), sendo necessária a titulação mínima de especialista. Embora os residentes tenham apontado a capacitação docente como uma das principais fragilidades, a dificuldade do controle e planejamento acadêmico sobre os preceptores, considerando que estes não pertencem às instituições que coordenam as RMS, também é uma realidade dos programas. $\mathrm{O}$ 
estudo de Wanderley (2010) ${ }^{21}$ aponta que a falta de preparo do preceptor para sua função é um ponto crucial na Residência Multiprofissional, estando diretamente relacionado com o comprometimento do processo de aprendizagem dos residentes.

De forma geral, a temática é bastante complexa, uma vez que envolve instituição formadora, executora, vários profissionais e suas individualidades. Fazer com que todos se integrem e atuem de maneira multi/ interprofissioanal é o maior desafio da RMS. Assim, considerando as limitações do presente estudo em relação à seleção das instituições de ensino e programas de RMS participantes, coleta e análise de dados, ressalta-se a necessidade da elaboração de novas pesquisas, utilizando outros métodos de coleta de informações e formas alternativas de coleta de dados, que auxiliem no aprimoramento da compreensão desse panorama.

\section{CONCLUSÕES}

O presente estudo possibilitou traçar um panorama do perfil profissional e da atuação de cirurgiões-dentistas inseridos em RMS da região Sul do Brasil. Os resultados caracterizam as práticas realizadas, o grau de satisfação dos cirurgiões-dentistas quanto às atividades e a organização das residências, assim como, as principais dificuldades encontradas nos programas de RMS.

Ressalta-se que a ausência de planejamento e estruturação pedagógica de algumas RMS, de alguma forma, reflete a falta de avaliação in loco dos programas existentes hoje no Brasil, considerando que todos os programas atualmente vigentes foram apenas autorizados. Sendo assim, o cumprimento das normas previstas nas várias resoluções publicadas pode acabar sem efeito.

Portanto, os dados encontrados no presente estudo podem auxiliar na melhoria das práticas e organização das RMS com cirurgiões-dentistas, conduzindo melhor a atuação de residentes, docentes, tutores e preceptores frente às principais fragilidades apontadas.

\section{ABSTRACT \\ Profile, performance, and satisfaction of Dentists in Multiprofessional Health Residences in Southern Brazil}

Multi-professional Health Residences (MHR) are distributed throughout the national territory and have an approach with a more human, comprehensive vision and whose focus is not restricted to therapeutic procedures. Therefore, the present study aimed to evaluate the professional profile of dentists in MHR in southern Brazil, as well as their performance and satisfaction with the residency program. Thus, quantitative, exploratory and descriptive research was carried out using an online questionnaire, whose link was sent to all dentists attending MHR in South Brazil. Subsequently, the data collected were tabulated in the Microsoft Excel ${ }^{\circledR}$ and treated by descriptive statistics analysis. Among the main results of personal and professional profiles, the majority of the residents were female $(75 \%)$ with a degree in private Higher Education Institutions (70\%), without prior professional experience $(70 \%)$ and with previous academic experience in Public health (85\%). For MHR data, the majority reported the interest in acting in the primary health care as a reason for choosing the residence. Concerning the place of performance, the Primary Health Unit represented 85\%. Most residents were satisfied with MHR (75\%) and reported the lack of teacher training as the main program weakness. Therefore, with this study, it was possible to draw a panorama of the professional profile and the performance of dentists inserted in MHR in Southern Brazil. Furthermore, data can support and improve the practices and organization of MHR with dentists, leading to better performance of residents and teachers in the main weaknesses pointed out.

Descriptors: Dentists. Family Health. Integrated Residency. Internship and Residency. 


\section{REFERÊNCIAS}

1. Brasil. Ministério da Saúde (MS). Secretaria de Atenção à Saúde. Departamento de Atenção Básica. Coordenação Nacional de Saúde Bucal. Diretrizes da Política Nacional de Saúde Bucal. Brasília: MS; 2004.

2. Rosa WAG, Labete RC. Programa Saúde da Família: a construção de um novo modelo de assistência. Rev. Latino-Am Enfermagem. 2005;13(6):1027-34.

3. Nascimento DDG, Oliveira MAC. Formação na Residência Multiprofissional em Saúde da Família. Saúde Social. 2010;19(4):814-817.

4. Brasil. Ministério da Educação. Resolução $\mathrm{n}^{\circ}$ 1, de 30 de janeiro de 2012. Comissão Nacional de Residência Multiprofissional. Institui as Câmaras Técnicas da Comissão Nacional de Residência Multiprofissional em Saúde e dá outras providências. Diário Oficial da União 31 jan. 2012; Seção 1.

5. MEC. Ministério da Educação e Cultura. Residência Multiprofissional [internet]. Brasília; 2016. [Acesso em 15 fev. 2017]. Disponível em: http://portal.mec.gov.br/resi dencias-em-saude/residencia-multiprofissional.

6. Pandolfi C. Utilização da pesquisa de satisfação de clientes como ferramenta para decisões gerenciais e melhoria contínua [dissertação]. Porto Alegre: Universidade Federal do Rio Grande do Sul; 2003.

7. SEMESP. Sindicato das Mantenedoras de Ensino Superior. Mapa do Ensino Superior no Brasil [internet]. São Paulo; 2015. [Acesso em 15 fev. 2017]. Disponível em: http://www.semesp.org.br/site/pesquisas/mapado-ensino-superior/mapa-do-ensino-superior2015/

8. Brasil. CNE. Resolução CNE/CES 3/2002 [internet]. Diário Oficial da União, Seção 1, p.10; Brasília, 4 de março de 2002. [Acesso em 15 fev. 2017]. Disponível em:
http://portal.mec.gov.br/cne/arquivos/pdf/C ES032002.pdf.

9. Bulgarelli AFB, Souza KR, Baumgarten A, Souza JM, Rosing CK, Toasse RFC. Formação em saúde com vivência no Sistema Único de Saúde (SUS): percepções de estudantes do curso de odontologia da UFRGS, Brasil. Interface (Botucatu). 2014;18(49):351-362.

10. Bottan ER, Vitoretti AJ, Neto UM. Perfil profissional do cirurgião-dentista em atuação no serviço público: a visão de um grupo de cirurgiões-dentistas. $\mathrm{R}$ Bras $\mathrm{Ci}$ Saúde. 2014;12(40):42-47.

11. Silva BS, Caldarelli PG. O PET-Saúde em consonância com as Diretrizes Curriculares Nacionais de Odontologia no desenvolvimento de competências profissionais: relato de experiência. Rev ABENO. 2013;13(2):34-41.

12. Saliba NA, Moimaz SAS, Prado RL, Garbin CAS. Percepção do cirurgião-dentista sobre formação profissional e dificuldades de inserção no mercado de trabalho. Rev Odont UNESP. 2012;41(5):297-304.

13. Fiocruz. Fundação Oswaldo Cruz. Pesquisa inédita traça perfil da enfermagem no Brasil [internet]. Rio de Janeiro; 2016. [Acesso em 15 fev. 2017]. Disponível em: http://portal.fiocruz.br/pt-br/content/pesquisainedita-traca-perfil-da-enfermagem-no-brasil.

14. Brasil. Resolução $n^{\circ} 5$, de 7 de novembro de 2014. Comissão Nacional de Residência Multiprofissional em Saúde - CNRMS. Dispõe sobre a duração e a carga horária dos programas de Residência em Área Profissional da Saúde nas modalidades multiprofissional e uniprofissional e sobre a avaliação e a frequência dos profissionais da saúde residentes. Diário Oficial da União 10 nov 2014; Seção 1.

15. Silva JC, Contim D, Ohl RIB, Chavaglia 
SRR, Amaral SEM. Percepção dos residentes sobre sua atuação no programa de RM. Acta Paul Enferm. 2015;28(2):132-38.

16. Alves J, Carvalho P. Importância do programa de Residência Multiprofissional em Saúde da Família da Universidade Federal de Pernambuco na formação dos profissionais da área da saúde. EFDesportes.com: Revista Digital. 2014, ano 18, n. 188.

17. Souza LEPF. Saúde Pública ou Saúde Coletiva? Revista Espaço para a Saúde. 2014;15(4):07-21.

18. Nascimento DDG, Oliveira MAC. A política de formação de profissionais da saúde para o SUS: considerações sobre a RMSF. REMERev Min Enferm. 2006;10(4):435-439.
19. Villalba JP, Madureira PR, Barros NF. Perfil profissional do cirurgião-dentista para atuação no Sistema Único de Saúde (SUS). Rev Inst Ciênc Saúde 2009;27(3):262-268.

20. Mello ALSF, Moyses ST, Moyses SJ. A universidade promotora de saúde e as mudanças na formação profissional. Interface (Botucatu). 2010;14(34):683-692.

21. Wanderley LCS. O processo de formação dos cirurgiões-dentistas da residência multiprofissional em saúde da família da Casa de Saúde Santa Marcelina: percepção do egresso [dissertação]. São Paulo: Faculdade de Odontologia, Universidade de São Paulo; 2010.

Correspondência para:

Pablo Guilherme Caldarelli

e-mail: pablocaldarelli@ hotmail.com

Rua Juiz de Fora, ${ }^{\circ}$ 230, Jardim Champagnat 86062-680, Londrina, PR 